\title{
Single and Double Photoionization from Dipole Response Function
}

\section{Citation}

Forrey, Robert C., Zong-Chao Yan, H. R. Sadeghpour, and A. Dalgarno. 1997. “Single and Double Photoionization from Dipole Response Function." Physical Review Letters 78 (19): 3662-64. https://doi.org/10.1103/physrevlett.78.3662.

\section{Permanent link}

http://nrs.harvard.edu/urn-3:HUL.InstRepos:41417270

\section{Terms of Use}

This article was downloaded from Harvard University's DASH repository, and is made available under the terms and conditions applicable to Other Posted Material, as set forth at http:// nrs.harvard.edu/urn-3:HUL.InstRepos:dash.current.terms-of-use\#LAA

\section{Share Your Story}

The Harvard community has made this article openly available.

Please share how this access benefits you. Submit a story.

Accessibility 


\title{
Single and Double Photoionization from Dipole Response Function
}

\author{
Robert C. Forrey, Zong-Chao Yan, H. R. Sadeghpour, and A. Dalgarno \\ Institute for Theoretical Atomic and Molecular Physics, Harvard-Smithsonian Center for Astrophysics, \\ 60 Garden Street, Cambridge, Massachusetts 02138
}

(Received 17 December 1996)

\begin{abstract}
A method for including the correlated motion of the electrons in the calculation of single and double ionization of atomic and molecular systems by the absorption of a single photon is described. The correlated dipole response function is central to the formulation. The single and double photoionization cross sections of helium are calculated. We show that the method accurately corrects for the error that is present at high energies when the length gauge is used to characterize the photon interaction. The cross sections obtained from the length, velocity, and acceleration gauges are brought into agreement with each other and with cross sections calculated from the many-body perturbation theory. [S0031-9007(97)03175-X]
\end{abstract}

PACS numbers: $32.80 . \mathrm{Fb}$

A central problem in theoretical atomic and molecular physics is the inclusion of the correlated motion of the electrons of the system. Much attention [1] has been given to the double ionization of helium by a single photon because the process occurs only through the effects of correlation. Taking correlation into account in the description of the final continuum state wave function presents severe difficulties. Several approaches have been pursued, including the many-body perturbation theory [24], close-coupling [5,6], and $R$-matrix procedures $[7,8]$ with a discretization of the continuum. Others [9-13] have employed explicit distorted wave approximations to the final state wave function.

In this Letter, we explore the applicability of a formulation in which the emphasis is placed on the inclusion of correlation in the dipole response function of the system. The method is then similar in physical content to the previous studies, but has the advantage that the difficulties associated with final state correlation can be handled using well-established $L^{2}$ methods. The same $L^{2}$ methods can also be used to describe correlation in the initial state wave function. The accuracy of the calculated cross sections is then limited only by the convergence of the $L^{2}$ basis sets. In the present work, we employ correlated Hylleraas basis sets which are known to converge quickly [14]. We carry out calculations in the length, velocity, and acceleration gauges. Previous calculations [6,7] have demonstrated that the length gauge is prone to error at high frequencies [15]. We show that our procedure converges rapidly and brings the results obtained with the different gauges into agreement.

The photoionization cross section of a photon with energy $\hbar \omega$ is given in atomic units in terms of the dipole matrix element by the expression

$$
\sigma=4 \pi^{2} \alpha \omega\left|\left\langle\Psi_{f}^{-}|\hat{\mathbf{e}} \cdot \mathbf{d}| \Psi_{i}\right\rangle\right|^{2},
$$

where $\Psi_{i}$ is the initial wave function, $\Psi_{f}^{-}$is the final wave function, $\mathbf{d}$ is the dipole moment, and $\hat{\mathbf{e}}$ is the direction of linearly polarized light. If $\mathbf{r}_{1}$ and $\mathbf{r}_{2}$ are the position vectors of the two electrons and $\mathbf{p}_{1}$ and $\mathbf{p}_{2}$ their momenta, the dipole moment can be expressed in the length, velocity, and acceleration gauges in the forms

$$
\begin{gathered}
\mathbf{d}_{L}=\mathbf{r}_{1}+\mathbf{r}_{2}, \quad \mathbf{d}_{V}=i \omega^{-1}\left(\mathbf{p}_{1}+\mathbf{p}_{2}\right), \\
\mathbf{d}_{A}=Z \omega^{-2}\left(\frac{\mathbf{r}_{1}}{\mathbf{r}_{1}^{3}}+\frac{\mathbf{r}_{2}}{\mathbf{r}_{2}^{3}}\right),
\end{gathered}
$$

where $Z$ is the nuclear charge. In the case of single ionization, the final state wave function describes the process $\hbar \omega+\mathrm{He} \rightarrow \mathrm{He}^{+}(n \ell)+e^{-}\left(\varepsilon^{\prime} \ell^{\prime}\right)$, where $n$ and $\ell$ are the quantum numbers of the final state of $\mathrm{He}^{+}$, and $\varepsilon^{\prime}$ and $\ell^{\prime}$ are the energy and angular momentum of the ejected electron. In double ionization $\hbar \omega+\mathrm{He} \rightarrow$ $\mathrm{He}^{2+}+e^{-}(\varepsilon \ell)+e^{-}\left(\varepsilon^{\prime} \ell^{\prime}\right)$, where $\varepsilon$ and $\varepsilon^{\prime}$ are the energies of the ejected electrons and $\ell$ and $\ell^{\prime}$ are their respective angular momenta. In the limit of high photon energies, only $\ell=0$ and $\ell^{\prime}=1$ contribute to the cross sections [16].

The final state wave function $\Psi_{f}^{-}$may be written

$$
\left|\Psi_{f}^{-}\right\rangle=\left|\psi_{f}^{-}\right\rangle+\frac{1}{E-i \eta-H} V\left|\psi_{f}^{-}\right\rangle,
$$

in which $E$ is the total energy of the system, $H=H_{0}+$ $V$ is the system Hamiltonian, and $\left|\psi_{f}^{-}\right\rangle$is the solution in the absence of the interaction potential $V$ between the separated systems. The dipole matrix element is [17]

$$
\left\langle\Psi_{i}|d| \Psi_{f}^{-}\right\rangle=\left\langle\Psi_{i}|d| \psi_{f}^{-}\right\rangle+\left\langle\chi|V| \psi_{f}^{-}\right\rangle,
$$

where

$$
|\chi\rangle=\frac{1}{E+i \eta-H} d\left|\Psi_{i}\right\rangle
$$

defines the dipole response function. If we represent $|\chi\rangle$ by an expansion in a discrete basis $\left|\theta_{m}\right\rangle$, such that $\left\langle\theta_{m}|H| \theta_{m^{\prime}}\right\rangle=E_{m} \delta_{m m^{\prime}}$, then

$$
|\chi\rangle=\sum_{m} \frac{\left|\theta_{m}\right\rangle\left\langle\theta_{m}|d| \Psi_{i}\right\rangle}{E-E_{m}} .
$$


With this choice for $|\chi\rangle$, the difficulties associated with the final state correlation can be handled using wellestablished bound-state $L^{2}$ procedures. In the present formulation, basis functions $\left|\theta_{m}\right\rangle$ are comprised of correlated Hylleraas-type functions [14].

We describe the asymptotic scattering solution corresponding to simultaneous excitation and ionization to the state $\left(n \ell, \varepsilon^{\prime} \ell^{\prime}\right)$ by the uncorrelated product state function

$$
\psi_{f}^{-}\left(\mathbf{r}_{1}, \mathbf{r}_{2}\right)=\frac{1}{\sqrt{2}}\left[u_{n \ell}\left(\mathbf{r}_{1}\right) F_{\varepsilon^{\prime} \ell^{\prime}}\left(\mathbf{r}_{2}\right)+u_{n \ell}\left(\mathbf{r}_{2}\right) F_{\varepsilon^{\prime} \ell^{\prime}}\left(\mathbf{r}_{1}\right)\right]
$$

where $F_{\varepsilon^{\prime} \ell^{\prime}}$ is an energy-normalized regular Coulomb function and $u_{n \ell}$ is an eigenfunction obtained by diagonalizing the single electron Hamiltonian in a 25 -term Sturmian basis set. The energy $\varepsilon^{\prime}$ that specifies the Coulomb function is obtained from $E=\varepsilon^{\prime}+\varepsilon_{n}$, where $\varepsilon_{n}$ is the eigenvalue associated with $u_{n \ell}$. Heller, Reinhardt, and Yamani [18] have shown that the use of a Sturmian basis set is equivalent to performing a Gaussian quadrature of the energy spectrum. Therefore, it is possible [6] to write for the total cross section for single ionization by a photon of energy $\hbar \omega$,

$$
\sigma^{+}=\sum_{n=0}^{n_{0}-1} \sum_{\ell, \ell^{\prime}} \sigma\left(n \ell, \varepsilon^{\prime} \ell^{\prime}\right)
$$

and for the cross section for double ionization

$$
\sigma^{2+}=\sum_{n=n_{0}}^{N} \sum_{\ell, \ell^{\prime}} \sigma\left(n \ell, \varepsilon^{\prime} \ell^{\prime}\right)
$$

where $N+1$ is the total number of terms in the Sturmian basis set and $n_{0}$ is the index corresponding to the lowest positive eigenvalue $\varepsilon_{n}$.

The initial state wave function was expanded in a correlated Hylleraas basis set [14]

$$
\phi=\left(1+\mathcal{P}_{12}\right) r_{1}^{a} r_{2}^{b} r_{12}^{c} \exp \left(-\alpha r_{1}-\beta r_{2}\right) Y_{\ell_{1} \ell_{2}}^{L M}\left(\hat{\mathbf{r}}_{1}, \hat{\mathbf{r}}_{2}\right),
$$

where $Y_{\ell_{1} \ell_{2}}^{L M}$ are total angular momentum eigenfunctions with orbital momentum $L$ and magnetic quantum number $M$, and $\mathcal{P}_{12}$ is the antisymmetrization operator. In (10) all terms are included such that $a+b+c \leq \Omega$. The parameters $\alpha$ and $\beta$ were optimized in order to obtain the lowest eigenvalue. To test for sensitivity to the initial state wave function, we varied $\Omega$ from 1 to 6 yielding eigenvalues ranging in accuracy from $10^{-3}$ to $10^{-9}$. We found that the cross sections are very sensitive to the representation of $\Psi_{i}$ when calculated in the length and velocity gauges, and are least sensitive in the acceleration gauge. For the dipole response function (6) we retained 112 terms in the correlated Hylleraas basis set (10) with the appropriate singlet $P$ symmetry. We restricted our calculations to high energies where we may assume $\ell=0$ and $\ell^{\prime}=1$. At lower energies, higher partial waves would need to be included.
The total cross sections obtained with the length formulation using the first term of expression (4) and using both terms for single and double ionization are compared in Fig. 1. Also included in Fig. 1 are the many-body perturbation theory results of Hino et al. [3]. The close agreement between our results and the results of the many-body perturbation theory suggests that the dipole response function has corrected the error associated with using only the first term in (4). In similar calculations using the velocity and acceleration gauges, the contribution from the dipole response term is small, and accurate results are obtained using only the first term. The different behavior of the three gauges can be understood by the perturbation theory analysis of Dalgarno and Lewis [19].

The method also yields reliable values of the cross sections for simultaneous excitation and ionization. The ratios of the $1 s, 2 s, 3 s$, and $4 s$ cross sections to the total cross sections are independent of energy above $1 \mathrm{keV}$ and equal to the respective values $0.9296,0.0446,0.0055$, and 0.0018 obtained from the asymptotic formulation of Dalgarno and Stewart $[16,20]$. The ratio of double to single photoionization cross section is nearly constant above $2 \mathrm{keV}$, consistent with the precise asymptotic value of $1.64 \%$ obtained by Forrey et al. [20].

Figure 1 shows that the representation of the final state wave function in (7) is inadequate at low energies and our cross sections lose accuracy. Preliminary calculations on a model problem suggest that the method will be successful at low energies provided we use the correct asymptotic form in (3). We are modifying our computational procedures to include the irregular Coulomb function in a distorted wave treatment that can be used at low energies.

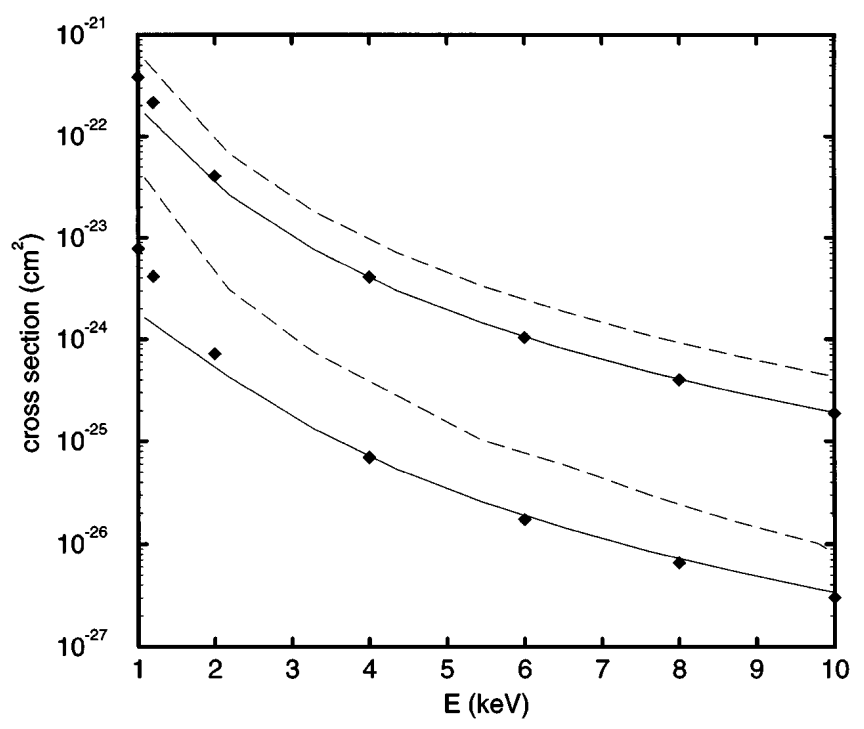

FIG. 1. The cross sections for single and double ionization of helium by a single photon. The dashed curves are calculated in the length gauge with the first term of (4) and the solid curves with both terms. The upper two curves refer to $\sigma^{+}$ and the lower two to $\sigma^{2+}$. The diamonds are the many-body perturbation theory results of Hino et al. [3]. 
This work was supported by the Division of Chemical Sciences, Office of Basic Energy Sciences, Office of Energy Research, U.S. Department of Energy. Z.C. Y. is also supported by the Natural Sciences and Engineering Research Council of Canada. The Institute for Theoretical Atomic and Molecular Physics at Harvard University and Smithsonian Astrophysical Observatory is supported by grants from the National Science Foundation.

[1] For a review of theory, see H. R. Sadeghpour, Can. J. Phys. 74, 727 (1996).

[2] S. L. Carter and H. P. Kelly, Phys. Rev. A 24, 170 (1981).

[3] K. Hino, T. Ishihara, F. Shimizu, N. Toshima, and J.H. McGuire, Phys. Rev. A 48, 1271 (1993).

[4] K. Hino, P. M. Bergstrom, Jr., and J. H. Macek, Phys. Rev. Lett. 72, 1620 (1994).

[5] J.-Z. Tang and I. Shimamura, Phys. Rev. 52, R3413 (1995).

[6] A. S. Kheifets and I. Bray, Phys. Rev. A 54, R995 (1996).

[7] K. W. Meyer and C. H. Greene, Phys. Rev. A 50, R3573 (1994).

[8] K. W. Meyer, C. H. Greene, and I. Bray, Phys. Rev. A 52, 1334 (1995).

[9] D. Proulx and R. Shakeshaft, Phys. Rev. A 48, R875 (1993).
[10] M. Pont and R. Shakeshaft, Phys. Rev. A 51, R2676 (1995).

[11] L. R. Andersson and J. Burgdörfer, Phys. Rev. Lett. 71, 50 (1993).

[12] M. A. Kornberg and J. E. Miraglia, Phys. Rev. A 48, 3714 (1993); 49, 5120 (1994).

[13] F. Maulbetsch and J. S. Briggs, J. Phys. B 26, 1679 (1993); 26, L647 (1993); 27, 4095 (1994).

[14] G. W. F. Drake, in Long-Range Casimir Forces: Theory and Recent Experiments on Atomic Systems, edited by F. S. Levin and D. A. Micha (Plenum, New York, 1993); Z.-C. Yan and G. W.F. Drake, Chem. Phys. Lett. 259, 96 (1996).

[15] A. Dalgarno and H.R. Sadeghpour, Phys. Rev. A 46, R3591 (1992).

[16] A. Dalgarno and A.L. Stewart, Proc. Phys. Soc. London 76, 49 (1960).

[17] A. Dalgarno, H. Doyle, and M. Oppenheimer, Phys. Rev. Lett. 29, 1051 (1972); H. Doyle, M. Oppenheimer, and A. Dalgarno, Phys. Rev. A 11, 909 (1975).

[18] E. J. Heller, W.P. Reinhardt, and H. A. Yamani, J. Comput. Phys. 13, 536 (1973); H. A. Yamani and W.P. Reinhardt, Phys. Rev. A 11, 1144 (1975).

[19] A. Dalgarno and J. T. Lewis, Proc. Phys. Soc. London Sect. A 69, 285 (1956).

[20] R. C. Forrey, H. R. Sadeghpour, J. D. Baker, J. D. Morgan III, and A. Dalgarno, Phys. Rev. A 51, 2112 (1995). 